\title{
Principales indicadores de Internet en la sociedad mexicana
}

\section{The Main Internet Indicators in Mexican Society}

Octavio Islas ${ }^{1}$

Amaia Arribas ${ }^{2}$

\section{Resumen}

El artículo explica qué es el World Internet Project (WIP) y por qué resulta tan relevante la iniciativa emprendida por el doctor Jeffrey Cole. Asimismo, se comparan algunos de los resultados que arrojó el estudio del WIP-México en 2009, con los resultados que presentan algunas de las principales investigaciones sobre Internet en México. En algunos apartados decidimos presentar la información que arrojaron algunas de estas investigaciones, sin considerar los resultados del estudio del WIP-México, por no haber considerado esas variables, pero pretendiendo ofrecer una perspectiva amplia del desarrollo que presenta Internet en México.

Palabras clave: World Internet Project, investigación usuarios de Internet en México, World Internet ProjectMéxico.
A Jerónimo. Infatigable guerrero del imaginario audiovisual.

\begin{abstract}
The article explains what the World Internet Project (WIP) is and why Dr. Jeffrey Cole's initiative is so relevant. It also compares some of the results of the WIP-Mexico study in 2009 to the results of several major research projects on the Internet in Mexico. In several sections, the authors present information from some of these studies, without taking into account the results of WIP-Mexico, which did not focus on the same variables. The idea is to offer a broad perspective of how the Internet has developed in the country.
\end{abstract}

Key words: World Internet Project, research on Internet users in Mexico, World Internet Project-México.

Recibido: 31/07/09

Reenviado: $19 / 05 / 10$

Aceptado: 27/10/10 


\section{Introducción}

En la primera parte del texto se explica qué es el World Internet Project (WIP), iniciativa del doctor Jeffrey Cole, director del Center for the Digital Future, de la Universidad del Sur de California en Annenberg, Estados Unidos, y se explica la relevancia de esa iniciativa. A partir de 2008, el estudio del WIP capítulo México es realizado por un grupo de investigadores adscritos al Proyecto Internet-Cátedra de Comunicaciones Digitales Estratégicas del Tecnológico de Monterrey, campus Estado de México (ITESM CEM). El estudio del WIP-Colombia lo llevan a cabo investigadores del Centro de Investigación de las Telecomunicaciones (Ceintel), con sede en Bogotá. Enseguida se indican los principales atributos metodológicos que permiten distinguir el estudio del WIP de investigaciones similares sobre la penetración de Internet, y se señalan las principales variables consideradas en el estudio.

En la segunda parte se comparan algunos de los resultados que arrojó el estudio del WIP-México en 2009, con los resultados que presentan algunos de los principales estudios sobre Internet en México, como el realizado por la Asociación Mexicana de Internet (AMIPCI), el de la firma Millward Brown para la Interactive Bureau (IAB), ComScore, Instituto Nacional de Estadística Geografía e Informática (INEGI), entre otros. Las variables contempladas en el análisis comparativo fueron: total de usuarios y penetración de Internet en México; razones expuestas por los no usuarios de Internet; distribución de los usuarios de Internet por género, edad y nivel socioeconómico; puntos de acceso a Internet; actividades sociales y consumo cultural; tecnologías que extienden a Internet; los usuarios ante el imaginario de Internet 2.0; cifras de Internet como medio publicitario en México, y México ante el imaginario de la sociedad de la información y el conocimiento.
Es importante señalar que la información consignada en algunos apartados de la segunda parte del texto no procede solo del estudio realizado por el WIP-México. No obstante, consideramos indispensable incorporar esa información en el texto, para ofrecer a los lectores de Palabra Clave una perspectiva más completa del impacto cultural de Internet en México.

\section{EI World Internet Project}

El World Internet Project (WIP) es una iniciativa del doctor Jeffrey Cole, destacado investigador estadounidense, director del Center for the Digital Future de la Annenberg School for Communication en la Universidad del Sur de California en Annenberg, Estados Unidos. El razonamiento que dio origen a la iniciativa del doctor Cole resulta tan contundente como sencillo. En los inicios de la televisión, no pocos investigadores de las ciencias sociales despreciaron investigar sobre televisión por considerarla como un tema poco relevante en el imaginario de los estudios de las ciencias sociales. Por supuesto, se cometió un grave error y perdimos la oportunidad de comprender mejor los efectos de la televisión estudiándola desde sus inicios. En no pocas ocasiones Jeffrey Cole ha sostenido que con el paso de los años Internet se convertirá en un medio aún más importante que la televisión. El argumento por lo menos admite ser considerado como una atractiva hipótesis de investigación.

Actualmente, reconocidos grupos de investigadores establecidos en 28 países se han sumado al World Internet Project para estudiar los efectos de la penetración de Internet en sus respectivas sociedades. En algunos países incluso participan equipos de investigadores de varias instituciones. La relación completa de los equipos de investigación participantes es la siguiente: Alemania (Deutsches Digital Institut), Argentina (Fundación de Investigaciones Económicas Latinoamericanas), Australia (Centre of Excellen- 
Volumen 13 Número 2 • Diciembre de 2010

ce for Creative Industries and Innovation y la Swinburne University of Technology), Bolivia (Universidad NUR), Canadá (Canadian Internet Project / Recherche Internet Canada), Chile (School of Communications Universidad Católica de Chile), China (Chinese Academy of Social Sciences), Chipre (Cyprus University of Technology Faculty of Applied Arts and Communication, Department of Communication and Internet Studies), Colombia (Centro de Investigación de las Telecomunicaciones), Corea del Sur (Yonsei University), Emiratos Árabes Unidos (Mass Communication Department-American University of Sharjah), España (Internet Interdisciplinary Institute y Open University of Catalonia), Estados Unidos (Center for the Digital Future USC Annenberg School for Communication), Francia (Center for Political Research), Hungría (ITHAKA- Information Society and Network Research Center), Irán (Faculty of Social Sciences and Economics University of Alzahra), Israel (The Research Center for Internet Psychology), Italia (SDA Bocconi, Bocconi University), Japón (Department of Media and Communications, Toyo University, The World Internet Project Japan), Macao (University of Macao), México (Proyecto Internet-Cátedra de Comunicaciones Digitales Estratégicas, ITESM CEM), Nueva Zelanda (Institute of Culture, Discourse \& Communication), Portugal (Lisbon Internet and Networks International Research Programme), Reino Unido (Oxford Internet Institute, University of Oxford), República Checa (Faculty of Social Studies Masaryk University in Brno), Rusia (Analytical Center, Video International), Singapur (Singapore Internet Research Centre), Suecia (World Internet Institute).

Estas son algunas de las principales diferencias que podemos advertir entre el estudio realizado por el World Internet Project y otras investigaciones sobre los usuarios de Internet:

1. El estudio del WIP centra su atención en el impacto social de Internet.
Actualmente, reconocidos grupos de investigadores establecidos en $\mathbf{2 8}$

países se han sumado al World Internet

Project para estudiar los efectos de la penetración de Internet en sus respectivas sociedades. En algunos países incluso participan equipos de investigadores de varias instituciones.

2. El proyecto repara en la necesidad de encuestar tanto a usuarios como a no usuarios de Internet.

3. Se trata de una iniciativa internacional.

4. Se desea obtener información indispensable para ofrecer una interpretación del proceso en desarrollo.

5. El principal objetivo del estudio es obtener información relevante para que el gobierno y la industria adopten políticas acertadas en materia de Internet.

El estudio del WIP comprende más de cien variables y la encuesta debe ser aplicada a los mismos informantes, pues se trata de una investigación longitudinal que repara en la necesidad de evaluar la evolución de los procesos analizados y el impacto de Internet en la calidad de vida de la población encuestada. Las principales variables consideradas en el estudio del WIP son:

1. Usuarios de Internet y no usuarios: ¿quién está en línea?, ¿quién no? ¿qué están haciendo los usuarios en línea?

- Acceso a Internet en casa, en la escuela y en el trabajo.

- Total de horas a la semana dedicadas a las principales actividades en la navegación en Internet.

- Años de acceso a Internet.

- Gasto destinado a la navegación en Internet. 
- Principales compras.

- Diferencias de las actividades que realizan en línea los usuarios experimentados y los nuevos usuarios.

- Porcentaje y distribución de las actividades realizadas semanalmente en Internet.

- Edades de los usuarios de Internet.

- Género.

- Uso de Internet en casa. Total de horas dedicadas a la navegación en Internet.

- Nivel socioeconómico.

- Tipo de conexiones a Internet en casa.

- Tipo de conexión - banda ancha o módem telefónico - y tipo de usuario - experimentado o nuevo-.

- Tipo de conexión y actividades en línea.

- Número de computadoras disponibles en casa y actividades que se realizan.

- Interfases y equipo electrónico avanzado disponible en casa - Personal Digital Assistant (PDAS) ${ }^{3}$, consolas de videojuegos, cámaras digitales-.

- Interfases y electrónica avanzada en usuarios experimentados y nuevos usuarios.

- Home networking.

- Número de computadoras destinadas a actividades de Home networking.

- Tiempo dedicado a blogs, publicar fotografías o mantener un sitio web personal.

- Tiempo dedicado a publicar información en Internet por edades.

- Contenido de lo que se publica en línea.

- No usuarios de Internet. ¿Por qué no usan Internet?

- ¿Por qué razones usuarios de Internet dejaron de serlo?

3 En castellano: asistente digital personal. Es una computadora de mano, originalmente diseñada como agenda electrónica e incluye calendario, lista de contactos, bloc de notas y recordatorios. Algunas disponen de algún sistema de reconocimiento de escritura. Actualmente los PDAS pueden realizar muchas de las funciones que hacen las computadoras de escritorio.
- ¿Qué han perdido al dejar de usar Internet?

- ¿Los no usuarios de Internet se volverán usuarios?

- Opinión ¿Cómo está transformando al mundo la tecnología?

- Lealtad de los usuarios a determinadas tecnologías (Internet, televisión, teléfonos celulares, etc.).

2. Uso del medio y confianza depositada

- ¿Por qué es tan importante Internet como medio de información?

- La importancia de Internet como medio informativo según nuevos usuarios y usuarios experimentados.

- La importancia de Internet como medio informativo según usuarios que establecen la conexión a través de módem telefónico y los usuarios que disponen de una conexión de banda ancha.

- Información en Internet. Credibilidad y actualidad.

- Pertinencia y calidad de la información disponible en los sitios más visitados.

- ¿Qué sitios en Internet son creíbles y cuáles no?

- Motores de búsqueda, ¿son confiables y su información se encuentra actualizada?

- Otras actividades multimedia que se realizan mientras se navega en Internet.

- Uso de Internet en la oficina.

- Uso de medios en línea - nuevos usuarios y usuarios experimentados-.

- Criterios de navegación.

- Popularidad de los sitios web.

- Motores de búsqueda, ¿están proporcionando la información que efectivamente buscan los usuarios?

3. Comportamiento como consumidores

- ¿Quién compra en línea?

- ¿Con qué frecuencia compra? 
- ¿Cuánto gasta?

- ¿Cuánto dinero destina a compras a través de Internet, compras por teléfono y compras por correo?

- Años de experiencia en la navegación en Internet y hábitos de compra.

- ¿Por qué los usuarios de Internet tardaron en realizar sus primeras compras en línea?

- Compras en línea, ¿cómo han incidido en los hábitos de compra en comercios establecidos?

- Búsqueda de productos y servicios -en línea y en comercios establecidos-.

- Búsqueda de productos y servicios -en línea y en comercios establecidos según nuevos usuarios y usuarios experimentados-.

- Actitudes acerca de las compras en línea.

- Actitudes acerca de la comercialización de servicios anteriormente gratuitos en Internet.

- Preocupación por temas de privacidad en usuarios que realizan compras en línea.

- Preocupación por temas de privacidad en no usuarios, nuevos usuarios y usuarios experimentados.

- Intención de pago por nuevos servicios.

- Preocupación por el empleo de tarjetas de crédito en operaciones en línea.

- Preocupación por el empleo de tarjetas de crédito en operaciones en línea - no usuarios, nuevos usuarios y usuarios experimentados-.

- Razones por las que expresan desconfianza al empleo de tarjetas de crédito en operaciones en línea.

- ¿Qué reduciría tal desconfianza? - usuarios experimentados y nuevos usuarios-.

4. Patrones de comunicación

- Uso del correo electrónico.

- Frecuencia con la cual consultan sus cuentas de correo.
- Reglas de etiqueta social en el manejo del correo electrónico.

- Prácticas de mensajería instantánea con más de una persona.

- Participación en comunidades en línea.

- Importancia concedida a la participación en comunidades en línea.

5. Efectos sociales

- ¿Afecta Internet tus relaciones con amigos y familiares?

- ¿Incrementa Internet tu contacto con personas con quienes compartes intereses?

- ¿Consideras que los usuarios de Internet pasan demasiado tiempo en línea?

- ¿Consideras que pasas demasiado tiempo en línea?

- Internet, los niños y el tiempo con los amigos.

- Internet y ver televisión, ¿cuánto tiempo deben dedicar a ello los niños?

- Tareas escolares y empleo de Internet.

- ¿La Internet contribuye a mejorar las notas escolares?

- Opinión sobre castigos que impiden ver televisión o usar Internet.

6. Poder político e influencia

- Participación y militancia política de los usuarios de Internet y los no usuarios.

- Importancia de Internet en el desarrollo de campañas políticas.

- Importancia de Internet en el desarrollo de campañas políticas según opiniones de usuarios y no usuarios.

- Internet y conocimiento de la actividad política.

- Internet y conocimiento de la actividad política (usuarios y no usuarios).

- Internet como herramienta para obtener ayuda política.

- Internet como herramienta para obtener poder.

- Información en línea y elecciones. 
- ¿En qué sitios los usuarios obtienen información sobre el desarrollo de campañas?

7. Internet en el trabajo

- Uso de Internet en el trabajo en horas de oficina.

- Uso de Internet en el trabajo en horas de oficina, usuarios nuevos y usuarios experimentados.

- Uso personal.

- ¿Internet hace más productivos a los trabajadores?

La investigación fue realizada en cada uno de los estados de la República y el Distrito Federal, en poblaciones con más de 50.000 habitantes. El trabajo de campo fue desarrollado durante los meses de octubre y noviembre de 2008, y el procesamiento de la información se efectuó durante el mes de diciembre de 2008. Vale la pena destacar que fueron realizadas 2.035 entrevistas entre usuarios y no usuarios de Internet en México mediante 27.310 llamadas telefónicas, que representan más de 35.000 minutos de tiempo aire. El error estándar del total de la muestra, considerando el universo por estudiar como infinito, con un intervalo de confianza de $95 \%$ y dando a las probabilidades de que ocurra o no el evento sus máximos valores (50 y 50\%) fue \pm $2,21 \%$. Además, debemos destacar que se observó la misma metodología que siguen cada uno de los equipos de investigadores en los países participantes en el WIP, incorporando algunas preguntas complementarias de particular interés para los patrocinadores (Microsoft, Prodigy MSN, HP, Procter \& Gamble).

\section{Principales indicadores relativos al desarrollo de Internet en México en 2009}

\section{Total de usuarios de Internet en México}

A finales de marzo de 2009, un grupo de investigadores adscritos al Proyecto Internet-Cátedra de Comunicaciones Digitales Estratégicas del ITESM CEM se dio a conocer los resultados que arrojó el "Estudio 2009 de hábitos y percepciones de los mexicanos sobre Internet y diversas tecnologías asociadas", primera investigación del WIP-México, la cual fue coordinada por Fernando Gutiérrez, director del Departamento de Comunicación del ITESM CEM e investigador en el Proyecto Internet-Cátedra de Comunicaciones Digitales Estratégicas. El estudio del WIP-México observó la misma metodología empleada por los grupos de investigadores asociados al World Internet Project en los 28 países que actualmente participan en la iniciativa de Jeffrey Cole. En nuestro estudio, el total de usuarios de Internet en México, de 12 a 70 años de edad, fue estimado en 25.217.500.

La tabla 1 presenta la información relativa al total de usuarios de Internet en México, estimados en 2007, 2008 y 2009 según los resultados que arrojaron algunos de los principales estudios sobre usuarios de Internet en México.

Tabla 1. Usuarios de Internet en México 2007, 2008 y 2009 (cifras en millones)

\begin{tabular}{|l|c|c|c|}
\hline \multicolumn{1}{|c|}{ Estudio } & $\mathbf{2 0 0 7}$ & $\mathbf{2 0 0 8}$ & $\mathbf{2 0 0 9}$ \\
\hline $\begin{array}{l}\text { Asociación Mexicana } \\
\text { de Internet (AMIPCI) }\end{array}$ & 23,7 & 27,6 & \\
\hline WIP-México & ---- & 25,2 \\
\hline $\begin{array}{l}\text { Comisión Nacional de } \\
\text { Telecomunicaciones } \\
\text { (Cofetel) }\end{array}$ & 22,1 & 23,3 & \\
\hline
\end{tabular}

Continúa

4 La Asociación Mexicana de Internet (Amipci) fue fundada en 1999 como Asociación Mexicana de la Industria Publicitaria y Comercial en Internet. Posteriormente cambió su nombre por Asociación Mexicana de Internet. De acuerdo con su misión - la cual se puede consultar en el sitio web de Ampici-, la Asociación Mexicana de Internet asume la obligación de: "potenciar la economía del Internet en México". La primera investigación de la Ampici sobre hábitos de los usuarios de Internet en México llevó el siguiente título: "Primer estudio de hábitos y tendencias en cibernautas mexicanos" y fue realizada por Greenfield Online, empresa estadounidense con represen- 


\begin{tabular}{|l|c|c|c|}
\hline \multicolumn{1}{|c|}{ Estudio } & $\mathbf{2 0 0 7}$ & $\mathbf{2 0 0 8}$ & $\mathbf{2 0 0 9}$ \\
\hline $\begin{array}{l}\text { comScore World } \\
\text { Metrix }\end{array}$ & 10,7 & 11,8 & \\
\hline eMarketer & 23,6 & 27,4 & \\
\hline $\begin{array}{l}\text { Instituto Nacional de } \\
\text { Estadística, Geografía } \\
\text { e Informática (Inegi) }\end{array}$ & 20.8 & 27,2 & \\
\hline $\begin{array}{l}\text { International } \\
\text { Telecommunication } \\
\text { Union (ITU) }\end{array}$ & 22.1 & ------ & ------ \\
\hline
\end{tabular}

Nota: *Mayores de 6 años, ** Mayores de 12 años, ${ }^{* * *}$ Mayores de 15 años

Fuente: Elaboración propia con base en la información resultante de estudios realizados por eMarketer: Mexico Online (2009) y WIP-México.

\section{Penetración de Internet en México}

Según información de la Internet World Stats ${ }^{6}$, la penetración de Internet en México se ubica entre las más bajas en la región $(24,8 \%)$-la penetración de Internet en Chile, por ejemplo, es superior al $50 \%$ - . La penetración de Internet en

tación en México. De acuerdo con David Fierro Villavicencio, citado por Islas et ál. (2002, p. 47): “El Comité de Investigación de Mercados de la Asociación Mexicana de Internet (Amipci) emprendió, en el año 2000, la primera investigación sobre los hábitos de los cibernautas en México. Tal esfuerzo representa el inicio de un compromiso periódico que nos permitirá identificar atractivas áreas de oportunidad para el desarrollo del comercio electrónico en nuestro país". La referida investigación -inexplicablemente ausente en el sitio web de Amipci - incluyó, según Villavicencio (citado por Islas et ál., 2002, p. 50): “los formatos de interceptación en línea, aleatorio y voluntario. El acceso a la encuesta se estableció a través de ventanas pop up (o contextuales) y banners (cintillos publicitarios que se publicaron en 15 sitios miembros de Ampici: Adnet, Burundis, CNI, El Sitio, Latinstocks, Palco Deportivo, Patagon, Reforma, Starmedia, T1msn, T02, UOL, Viajo, Vota Por y Yahoo". El "Estudio Amipci. Hábitos de los usuarios de Internet en México" fue dado a conocer en mayo de 2009. Los estudios realizados por la Amipci pueden ser consultados en http://www.amipci.org.mx/estudios/

5 En su estudio, la firma ComScore no consideró el acceso a Internet desde sitios públicos (cibercafés, por ejemplo), excluyendo además el acceso a Internet a través de teléfonos móviles o PDA. No obstante, en una presentación disponible en http://www.scribd.com/ doc/15485661/ComScore-State-Of-Internet-Mexico-Apr09, en la pantalla número 7 Jonathan Álvarez - ejecutivo de ComScore - aseveró que esa firma reconoce 24.7 millones de usuarios de Internet en México, según el "universo extendido", en el cual fueron incluidos menores de 15 años y personas que comparten una misma computadora.

6 Los datos fueron registrados por la Internet World Stats el 30 de septiembre de 2009.
México resulta $0,8 \%$ inferior al promedio mundial $(25,6 \%)$, y 5,7\% inferior al promedio registrado en América Latina y el Caribe (30,5\%). La diferencia entre el dato ofrecido por la Internet World Stats $(24,8 \%)$ y el dato que arrojó el estudio del WIP-México (23,6\%) apenas varía 1,2\%. En la tabla 2 hemos incluido las estimaciones relativas a la penetración de Internet en México, consignadas en los estudios anteriormente referidos. La cifra presentada por la Amipci $(29,7 \%)$, por ejemplo, se dispara sensiblemente del dato que consigna la Internet World Stats.

\section{Tabla 2. Penetración de Internet en México}

\begin{tabular}{|l|c|}
\hline \multicolumn{1}{|c|}{$\begin{array}{c}\text { Estudio y fecha de la presentación } \\
\text { de los resultados }\end{array}$} & Porcentaje \\
\hline $\begin{array}{l}\text { Asociación Mexicana de Internet (Amipci), } \\
\text { mayo de 2009* }\end{array}$ & 29,7 \\
eMarketer (enero de 2009) & 24,9 \\
Internet World Stats (septiembre 30 de 2009) & 24,8 \\
WIP** (marzo de 2009) & 23,6 \\
International Telecommunication Union (ITU) (2008) & 20,8 \\
comScore World Metrix (2008) & 14,0 \\
\hline
\end{tabular}

Nota: $\quad$ *Mayores de 15, **Proyección Nacional: Base: 106,7 millones de mexicanos. No fueron considerados menores de 12 ni mayores de 70 años.

Fuente: elaboración propia, con base en la información resultante de estudios realizados por eMarketer: Mexico Online (2009) y el WIP-México.

\section{Principales razones por las cuales los no usuarios de Internet no utilizan la Red}

En nuestra investigación contemplamos algunas preguntas a no usuarios de Internet. En la pregunta relativa a las razones por las cuales aún no utilizan la Red, los encuestados pudieron elegir varias opciones. Por esa razón la suma de las respuestas posibles es superior al $100 \%$. Estos fueron los resultados:

1. No le interesa: $31 \%$

2. No tiene computadora: $28 \%$ 
3. No sabe usarla: $24 \%$

4. No tiene tiempo: $15 \%$

5. Es muy caro: $12 \%$

6. Otras razones: $5 \%$

\section{Infraestructura tecnológica}

De acuerdo con lo asentado en el reciente estudio realizado por la Amipci, que recurre a investigaciones realizadas por Select como fuente de información en el apartado de infraestructura tecnológica, el total de computadoras en México fue estimado en 18,2 millones ${ }^{7}$. De ese total, solo 11,3 millones de computadoras disponían de la tecnología necesaria para permitir el acceso a Internet (62,8\%). Además, el 51\% de las computadoras con acceso a Internet se encontraban instaladas en los hogares y el 93\% de las cuentas de acceso a Internet eran de banda ancha. El total de teléfonos móviles en uso fue estimado en 73,6 millones. El promedio de computadoras por cada 10 hogares, se estimó en 3,5.

\section{Distribución de los usuarios de Internet en México por género}

Los principales estudios sobre los usuarios de Internet en México coinciden en ubicar la existencia de una marcada brecha por género en el acceso a Internet. De acuerdo con los resultados que arrojó el estudio realizado por el WIP-México, $58 \%$ de los usuarios de Internet son hombres y $42 \%$ son mujeres. Según el estudio que la Amipci presentó en mayo de 2009,56\% de los usuarios de Internet son hombres y $44 \%$ mujeres.

\section{Distribución de los usuarios de Internet en México por edad}

El reciente estudio de la Amipci presenta la siguiente información sobre la penetración de

7 En el estudio correspondiente a 2008 de la Amipci fueron estimadas 17,8 millones de computadoras en México.
Internet según las edades de los usuarios de Internet (tabla 3$)^{8}$ :

Tabla 3. Penetración de Internet

considerando las edades de los usuarios

\begin{tabular}{|c|c|c|}
\hline Edad & $\begin{array}{c}\text { Penetración del universo } \\
\text { de internautas por edad (\%) }\end{array}$ & $\begin{array}{c}\text { Total relativo a la } \\
\text { población nacional (\%) }\end{array}$ \\
\hline $12-19$ & 63 & 22 \\
$20-24$ & 55 & 14 \\
$25-34$ & 35 & 25 \\
$35-44$ & 24 & 19 \\
$45-54$ & 19 & 13 \\
$55-64$ & 10 & 7 \\
\hline
\end{tabular}

Fuente: Amipci (2009).

\section{Distribución de los usuarios de Internet en México por nivel socioeconómico}

Según los resultados que arrojó nuestro estudio, los niveles socioeconómicos de los usuarios de Internet en México son los siguientes (tabla 4):

Tabla 4. Nivel socioeconómico de los usuarios de Internet en México, según el estudio del WIP-México

\begin{tabular}{|c|c|c|c|}
\hline $\begin{array}{c}\text { Niveles } \\
\text { socioeconómicos }\end{array}$ & $\begin{array}{c}\text { Porcentaje } \\
\text { de usuarios }\end{array}$ & $\begin{array}{c}\text { Porcentaje } \\
\text { no usuarios }\end{array}$ & $\begin{array}{c}\text { Porcentaje } \\
\text { relativo } \\
\text { considerando el } \\
\text { total de usuarios }\end{array}$ \\
\hline A,B, C+ & 57 & 33 & 41 \\
C & 45 & 55 & 22 \\
D+ & 30 & 70 & 28 \\
D/E & 21 & 79 & 9 \\
\hline
\end{tabular}

Fuente: World Internet Project México.

La tabla 5 presenta los resultados que arrojó el estudio de la Amipci en 2009:

8 La fuente que emplea la Amipci en el "Estudio Amipci. Hábitos de los usuarios de Internet en México" y, particularmente en el apartado "Perfil del Internauta Mexicano", procede de estudios realizados por TGI Kantar Media Research.

9 En el congreso de la Asociación Mexicana de Agencias de Investigación de Mercados y Opinión Pública (AMAI), celebrado el 9 de septiembre de 2008, Heriberto López Romo, destacado investigador mexicano, explicó las modificaciones al índice de nivel socioeconómico, conocido como Regla AMAI 13x6, que desde 1994 es empleado 
Tabla 5. Nivel socioeconómico de los usuarios de Internet en México, según el Estudio 2009 de hábitos y percepciones de los mexicanos sobre Internet $y$ diversas tecnologías asociadas

\begin{tabular}{|c|c|c|}
\hline $\begin{array}{c}\text { Niveles } \\
\text { socioeconómicos }\end{array}$ & $\begin{array}{c}\text { Porcentaje } \\
\text { de usuarios }\end{array}$ & Población total \\
\hline A,B, C+ & 63 & 22 \\
C & 43 & 17 \\
D+ & 33 & 33 \\
D/E & 20 & 29 \\
\hline
\end{tabular}

Fuente: Amipci (2009).

\section{Acceso a Internet}

De acuerdo con los resultados que arrojó nuestro estudio, el 69\% de los usuarios de Internet indicaron que su principal punto de conexión son los cibercafés, la casa de amigos y las bibliotecas; en segundo lugar mencionaron el hogar (61\%); en tercero la escuela (43\%), y el trabajo fue ubicado en la cuarta posición (28\%).

Según los resultados del estudio de la Amipci, el hogar es el principal punto de acceso a Internet $(48 \%)$, en segundo lugar los cafés Internet y sitios de acceso público (34\%), trabajo (19\%); escuela y universidad (11\%), casa de otras personas $(4 \%)$, otros $(1 \%)$.

De acuerdo con los resultados del Estudio de consumo de medios digitales en México, realizado por

como criterio de clasificación de la AMAI, para la investigación de mercados en México. La Regla 13x6 clasifica a los hogares mexicanos en seis niveles socioeconómicos, a partir de un árbol de asignaciones que considera, desde 2008, 10 nuevos indicadores: número de televisores en color y computadoras (tecnología y entretenimiento); número de focos, número de autos y estufa (infraestructura práctica); baños y regadera (infraestructura sanitaria); tipo de piso y número de habitaciones (infraestructura básica); capital humano (educación del jefe de familia). A las 10 variables referidas les asignaron puntos, considerando el coeficiente de cada uno de los valores en una regresión sobre el ingreso familiar. El nivel E - el menos favorecido- suma hasta 60 puntos; el nivel D, entre 61 y 101 puntos; el nivel D+, entre 102 y 156; el nivel C, entre 157 y 191; el nivel C+, entre 192 y 241; los niveles A/B entre 242 y más. Véase: http://www.amai.org/ congreso/2008/memorias/ponencias/lopezromo.pdf [Fecha de consulta: 22 de enero de 2010].
Millward Brown México para la IAB, la casa es el principal punto de acceso a Internet en México (41\%); en segundo lugar está el trabajo (36\%); en tercero los cafés Internet (11\%); lugar de estudio $(8 \%)$ y casa de otros $(4 \%)$.

El tiempo promedio diario de conexión a Internet, según el reciente estudio de la Amipci, es 2 horas 54 minutos. El tráfico más intenso se registra de las 16 a las 18 horas de lunes a viernes.

\section{Actividades sociales de los usuarios de Internet en México}

De acuerdo con el estudio del WIP-México, las principales actividades de los usuarios de Internet en México son:

- Revisar correo electrónico (93\%).

- Usar el MSN (83\%).

- Mandar documentos por correo electrónico $(83 \%)$.

- Trabajos de la escuela (80\%).

- Buscar noticias (78\%).

- Buscar definiciones (78\%).

- Bajar y escuchar música (77\%).

- Confirmar un hecho (75\%).

- Información sobre salud (73\%).

- Buscar bromas y caricaturas (48\%).

- Información de viajes (44\%).

- Leer blogs (42\%).

- Navegar sin quedarse en un sitio (41\%).

- Jugar (39\%).

- Chatear en foros (37\%).

El tiempo promedio diario de conexión a Internet, según el reciente estudio de la Amipci, es 2 horas 54 minutos. El tráfico más intenso se registra de las 16 a las 18 horas de lunes a viernes. 
- Trabajar en tu blog (33\%).

- Tener acceso a redes (32\%).

- Trámites fiscales $(29 \%)$.

- Escuchar radio en línea (28\%).

- Hacer o recibir llamadas (21\%).

- Participar en cursos (16\%).

- Visitar sitios peligrosos (14\%).

- Visitar sitios de contenido social (9\%).

- Visitar sitios de apuestas (3\%).

Con base en los resultados que arrojó nuestro estudio, podemos afirmar que en materia de prácticas comunicativas, el usuario de Internet en México se encuentra en la transición de los ambientes mediáticos de Internet - revisar correo, por ejemplo- a los propios de Internet 2.0 -tener acceso a redes, por ejemplo-. Según los resultados que arrojó el estudio de la Amipci, las principales actividades de los usuarios de Internet en México son:

- Enviar-recibir correo electrónico (70\%).

- Enviar-recibir mensajes instantáneos (58\%).

- Entrar en un chat room (41\%).

- Subir videos o fotos en un sitio para compartirlos (34\%).

- Enviar postales electrónicas (26\%).

- Crear / mantener blog / bitácora personal (16\%).

- Acceder a blog / bitácora personal (16\%).

- Crear / mantener sitio propio (15\%).

- Acceder a sitios de comunidad virtual (10\%).

En México los usuarios de Internet empiezan a asumir los roles comunicativos que distinguen a los prosumidores (Toffler), pues han optado por experimentar el tipo de prácticas comunicativas que posibilita el desarrollo de Internet 2.0.
- Encuentros online (10\%).

- Crear / mantener sitios de comunidad virtual (9\%).

Independientemente de las sensibles variaciones que podemos advertir en ambos estudios al considerar determinadas prácticas comunicativas, en términos generales el estudio realizado por la Amipci confirma las tendencias que logramos advertir al analizar los resultados que arrojó nuestro estudio: en México los usuarios de Internet empiezan a asumir los roles comunicativos que distinguen a los prosumidores (Toffler), pues han optado por experimentar el tipo de prácticas comunicativas que posibilita el desarrollo de Internet 2.0. Los estudios de Ibope y de la IAB confirman tales tendencias.

De acuerdo con los resultados que arrojó el Anuario 2008-2009. Audiencias y Medios en México, de febrero de 2009, Ibobe AGB México, las principales actividades de los usuarios de Internet en México son:

- Enviar o leer correos electrónicos (70\%).

- Enviar-recibir mensajes instantáneos (MSN), etc. $(58 \%)$.

- Entrar en un chat room (41\%).

- Subir fotos o videos en un sitio para compartirlos (34\%).

- Enviar postales electrónicas (25\%).

- Crear, mantener blog / bitácora personal (15\%).

- Accesar a blogs / bitácora personal (15\%).

- Crear / mantener un sitio propio (14\%).

- Accesar a sitios de comunidad virtual (10\%).

- Encuentros "online" (10\%).

- Crear / mantener sitios de comunidad virtual $(8 \%)$.

Según los resultados que arrojó el reciente estudio de la IAB, las principales actividades en 
línea de los usuarios de Internet, en general, y de los jóvenes, en particular, son las siguientes (tabla 6):

Tabla 6. Actividades en línea de los usuarios de Internet y de los jóvenes

\begin{tabular}{|l|c|c|}
\hline \multicolumn{1}{|c|}{ Actividad } & Usuarios & Jóvenes \\
\hline Enviar o revisar correos & 87 & 82 \\
Buscar información & 77 & 78 \\
Chatear / Mensajes instantáneos & 41 & 63 \\
Leer noticias en línea & 54 & 28 \\
\hline
\end{tabular}

Fuente: IAB (s. f.).

En la oferta cultural de Internet, la variable edad resulta determinante en la selección de contenidos. Los jóvenes esperan participar, situación que naturalmente los perfila como público meta en los ambientes comunicativos que desplaza consigo el desarrollo de Internet 2.0, destacando la blogósfera y la twittósfera.

El estudio de la IAB, además, reporta información sobre las principales actividades que los usuarios de Internet realizan de forma simultánea, es decir, mientras navegan en Internet. Tal fenómeno permite confirmar la pertinencia de las agudas observaciones relativas a las capacidades multitasking (multitarea) en los llamados "nativos digitales", realizadas por expertos como Piscitelli, Boschma, Islas y Gutiérrez (tabla 7).

Tabla 7. Actividades que realizaban de manera simultánea al navegar en Internet

\begin{tabular}{|l|c|}
\hline \multicolumn{1}{|c|}{ Actividad } & Usuarios (\%) \\
\hline Hablar por teléfono & 50 \\
Trabajar & 41 \\
Escuchar música & 36 \\
Ver televisión & 25 \\
Enviar mensajes de texto & 22 \\
\hline
\end{tabular}

Fuente: IAB (s. f.).
El reciente estudio de la Amipci aporta información relativa a las principales actividades de los usuarios de Internet en materia de entretenimiento:

- Bajar música (49\%).

- Ver chistes o páginas de humor (32\%).

- Jugar on line (30\%).

- Visitar sitios deportivos (28\%).

- Astrología, horóscopos (20\%).

- Otras actividades (18\%).

En el estudio de la Amipci también se consideró la forma como los usuarios de Internet emplean los medios de comunicación convencionales. La importancia del asunto radica en la posibilidad de confirmar algunas de las principales hipótesis formuladas en torno a las remediaciones ${ }^{10}$ en los medios. Las principales actividades en línea de los usuarios de Internet en México fueron:

10 En la ecología de los medios - la escuela en el imaginario de la comunicología posible que con mayor rigor ha abordado el tema del impacto de los cambios tecnológicos en las sociedades-, la columna vertebral de la ecología de los medios son las tesis de Marshall McLuhan, y es conocida también como Escuela de Toronto, Escuela de Nueva York, Escuela de San Luis, Escuela Norteamericana de la Comunicación, el concepto remediación admite por lo menos dos interpretaciones. En el libro The Soft Age, Paul Levinson (1997) empleó el término "medio remedial" para describir cómo nuestras sociedades utilizan un medio para reformar o mejorar a otro. De acuerdo con Levinson, en un esfuerzo que no siempre advertimos, intentamos hacer que los medios se parezcan más al hombre. En uno de sus recientes libros: Cellphone. The story of the world's most mobile medium and how it has transformed everything, Levinson afirmó que los medios de comunicación aún compiten -y de forma más intensa - por la atención de las personas (Levinson, 2004, p. 12). En términos darwinianos - sugiere Levinson - , la selección del ambiente mediático queda en manos de las personas, quienes contribuyen con su preferencia a la evolución de un determinado medio. Constantemente se decide entre ir al cine o quedarse en casa a ver televisión, leer un libro o ver un video, hablar por celular o enviar un correo electrónico. Los medios no evolucionan por una selección natural sino por una elección humana. El medio que mejor evoluciona es aquel que se ajusta más a las diversas necesidades del hombre. El proceso de remediación de los medios de comunicación sintetiza tal adecuación que les permite "imitar" las operaciones realizadas por las facultades y órganos del ser humano. Jay Bolter y Richard Grusin emplean el concepto remediación para describir las relaciones formales de interdependencia cultural que existen entre dos o varios medios, argumentando que en las primeras líneas de Comprender a los medios de comunicación: Las extensiones del ser humano, Marshall McLuhan afirmó que el contenido de todo medio es otro medio. Para Bolter y Grusin, Internet es un nuevo medio remediador, pues asimila a los que le antecedieron. El contenido de Internet son los medios que le antecedieron. 
- Leer diarios / periódicos (22\%).

- Escuchar radio (11\%).

- Ver programas de TV (8\%).

- Leer revistas (6\%).

El estudio realizado por Ibope aporta información relevante sobre el consumo cultural de los usuarios de Internet en México, analizando usos sociales: usos informativos, viajes y entretenimiento, medios de comunicación y finanzas, empleo, compras y búsqueda. Los principales usos informativos de Internet fueron:

- Realizar investigación personal (63\%).

- Visitar sitios de educación / aprendizaje (36\%).

- Visitar sitios de salud (25\%).

- Consultar sitios de películas de cine (23\%).

Asimismo, el estudio de Ibope repara en la utilidad de las funciones de entretenimiento que puede realizar Internet - desde la tradición Estructural Funcionalista, autores como Wright han reparado en la importancia de dichas funciones que pueden realizar los medios de comunicación-. Los principales usos de Internet en materia de entretenimiento fueron:

- Bajar música (49\%).

- Ver chistes o páginas de humor (31\%).

- Jugar online (30\%).

- Visitar sitios deportivos (28\%).

Los principales usos de Internet como medio de comunicación exhiben a un usuario de Internet promedio, el cual aún no ha advertido la posibilidad de asumir el papel de un activo gestor de contenidos en línea. Los principales usos de Internet como medio de comunicación son:

- Escuchar música (48\%).

- Leer noticias nacionales (34\%).

- Leer noticias internacionales (29\%).

- Leer diarios $(22 \%)$.
En el estudio realizado por Ibope fueron consideradas interesantes relaciones entre variables, por ejemplo, qué tipo de actividades culturales realiza el usuario en Internet y su nivel socioeconómico. Evidentemente, se pretende establecer la posible relación que observa el tipo de consumo cultural en Internet con la estratificación social de los usuarios. En los niveles A, B, C+ las principales actividades en Internet fueron:

1. Leer noticias internacionales.

2. Consultar directorios de negocios y empresas.

3. Leer noticias nacionales.

4. Buscar en clasificados casa, autos, etc.

En el extremo opuesto, en los niveles D y E, las principales actividades en Internet fueron:

1. Jugar online.

2. Entrar en un chat room.

3. Bajar música.

4. Escuchar música.

En cuanto a las principales actividades en Internet, considerando la variable género, las que recibieron el mayor número de menciones en hombres fueron:

1. Buscar en clasificados autos, personales, casas.

2. Consultar directorios de negocios y empresas.

3 Jugar online.

4. Leer noticias internacionales.

Las actividades que recibieron el mayor número de menciones en mujeres fueron:

1. Visitar sitios de salud.

2. Visitar sitios de educación / aprendizaje.

3. Enviar y recibir mensajes instantáneos.

4. Enviar y recibir correos electrónicos. 
La variable género admite pues particular relevancia en la oferta cultural de contenidos en Internet. El tipo de consumo cultural que distingue a las mujeres supone un mayor nivel de socialización.

Las principales actividades en línea varían significativamente al considerar la variable edad. En el rango de 12-19 años, las principales actividades en línea fueron:

1. Jugar a juegos online.

2. Ver chistes o páginas de humor.

3. Escuchar música.

En el rango de 20-24 años, las principales actividades en línea fueron:

1. Bajar música.

2. Enviar / recibir mensajes instantáneos.

3. Entrar en un chat room.

En el rango de 25-34 años, las principales actividades en línea fueron:

1. Leer noticias nacionales.

2. Leer noticias internacionales.

3. Visitar sitios de salud.

En el rango de 35-44 años, las principales actividades en línea fueron:

1. Leer noticias internacionales.

2. Visitar sitios de salud.

3. Leer noticias nacionales.

En el rango de 45 a 54 años, las principales actividades en línea fueron:

1. Consultar directorios de negocios y empresas.

2. Leer noticias internacionales.

3. Leer noticias nacionales.
En el rango de 55 a 64 años, las principales actividades en línea fueron:

1. Leer noticias internacionales.

2. Leer noticias nacionales.

3. Visitar sitios de salud.

\section{Disposición para el uso de nuevas tecnologías}

De acuerdo con los resultados que arrojó el estudio realizado por Millward Brown México para la IAB, la aceptación de determinadas tecnologías registró un sensible incremento con respecto a 2008 (tabla 8):

Tabla 8. Disposición para el uso de nuevas tecnologías

\begin{tabular}{|l|c|c|c|}
\hline Tecnología & 2008 (\%) & 2009 (\%) & Variación \\
\hline Celular & 86 & 88 & +2 \\
Computadora & 80 & 86 & +6 \\
Cámara digital & 62 & 70 & +8 \\
Reproductor MP3 & 54 & 60 & +6 \\
PDAs/Handhelds & 16 & 13 & -3 \\
\hline
\end{tabular}

Fuente: IAB (s. f.).

El papel que observa la tecnología en la ecología cultural de las sociedades no necesariamente es similar. En Japón, por ejemplo, el uso de la tecnología resulta natural y las series animadas dan cuenta de ello. En caricaturas como Giganthor (1964) los niños manejan las operaciones de enormes robots. En cambio, en no pocas naciones -Cuba, por ejemplo- es posible advertir sistemática desconfianza a los efectos de los cambios tecnológicos, los cuales suelen ser asociados con conspiraciones que atentan contra la cultura y que responden al apetito imperialista. Es posible afirmar que en años recientes la sociedad mexicana ha desarrollado mayor receptividad al uso de nuevas tecnologías. 


\section{Los usuarios de Internet en México frente a los medios de comunicación convencionales}

El estudio realizado por la IAB consideró tres interesantes variables en la relación de los usuarios de Internet con los medios de comunicación masiva convencionales: atributos emocionales, atributos del medio y atributos de relación. Veamos los resultados (tabla 9):

Tabla 9. Atributos emocionales

\begin{tabular}{|l|c|c|c|c|c|c|}
\hline & $\begin{array}{c}\text { Internet } \\
(\%)\end{array}$ & $\begin{array}{c}\text { Televisión } \\
(\%)\end{array}$ & $\begin{array}{c}\text { Radio } \\
(\%)\end{array}$ & $\begin{array}{c}\text { Revistas } \\
(\%)\end{array}$ & $\begin{array}{c}\text { Períódicos } \\
(\%)\end{array}$ & $\begin{array}{c}\text { Exteriores } \\
(\%)\end{array}$ \\
\hline & $2008-2009$ & $2008-2009$ & $2008-2009$ & $2008-2009$ & $2008-2009$ & $2008-2009$ \\
\hline $\begin{array}{l}\text { Disfruto } \\
\text { utilizarlo }\end{array}$ & $71-77$ & $43-47$ & $28-31$ & $21-31$ & $15-20$ & $3-4$ \\
$\begin{array}{l}\text { Es para alguien } \\
\text { de mi edad }\end{array}$ & $68-74$ & 4646 & $34-36$ & $31-40$ & $25-26$ & $13-14$ \\
$\begin{array}{l}\text { Indispensable } \\
\text { en mi vida }\end{array}$ & $48-54$ & $30-30$ & $19-22$ & $8-12$ & $11-15$ & $2-3$ \\
Lo amo & $34-41$ & $23-24$ & $14-15$ & $10-16$ & $5-8$ & $2-3$ \\
\hline
\end{tabular}

Fuente: IAB (s. f.).

En el periodo 2008-2009, en México mejoró la percepción de los usuarios de Internet encuestados sobre los atributos emocionales que suscitan los medios que fueron considerados en el estudio, destacando Internet, que registró los más altos niveles de satisfacción (tabla 10).

Tabla 10. Atributos del medio

\begin{tabular}{|l|c|c|c|c|c|c|}
\hline & $\begin{array}{c}\text { Internet } \\
(\%)\end{array}$ & $\begin{array}{c}\text { Televisión } \\
(\%)\end{array}$ & $\begin{array}{c}\text { Radio } \\
(\%)\end{array}$ & $\begin{array}{c}\text { Revistas } \\
(\%)\end{array}$ & $\begin{array}{c}\text { Periódicos } \\
(\%)\end{array}$ & $\begin{array}{c}\text { Exteriores } \\
(\%)\end{array}$ \\
\hline & $2008-2009$ & $2008-2009$ & $2008-2009$ & $2008-2009$ & $2008-2009$ & $2008-2009$ \\
\hline $\begin{array}{l}\text { Me mantiene } \\
\text { actualizado }\end{array}$ & $74-81$ & $42-45$ & $27-30$ & $19-25$ & $28-31$ & $5-8$ \\
$\begin{array}{l}\text { Estoy muy } \\
\text { expuesto a este } \\
\text { medio de } \\
\text { comunicación }\end{array}$ & $58-64$ & $48-50$ & $28-29$ & $13-18$ & $15-18$ & $16-22$ \\
$\begin{array}{l}\text { Marcan } \\
\text { tendencias }\end{array}$ & $50-58$ & $48-50$ & $16-16$ & $24-32$ & $9-12$ & $8-16$ \\
$\begin{array}{l}\text { Es el medio de } \\
\text { comunicación } \\
\text { más accesible }\end{array}$ & $38-45$ & $49-51$ & $35-32$ & $6-10$ & $14-19$ & $8-13$ \\
\hline
\end{tabular}

Fuente: IAB (s. f.).
En el periodo 2008-2009, en México también mejoró la percepción de los usuarios de Internet que fueron encuestados sobre los atributos de los medios, con excepción de la radio, que registró un inesperado descenso en términos de accesibilidad. De nueva cuenta vale la pena destacar los positivos y elevados porcentajes de satisfacción que suscitó Internet (tabla 11).

Tabla 11. Atributos de relación

\begin{tabular}{|l|c|c|c|c|c|c|}
\hline & $\begin{array}{c}\text { Internet } \\
(\%)\end{array}$ & $\begin{array}{c}\text { Televisión } \\
(\%)\end{array}$ & $\begin{array}{c}\text { Radio } \\
(\%)\end{array}$ & $\begin{array}{c}\text { Revistas } \\
(\%)\end{array}$ & $\begin{array}{c}\text { Periódicos } \\
(\%)\end{array}$ & $\begin{array}{c}\text { Exteriores } \\
(\%)\end{array}$ \\
\hline $2008-2009$ & $2008-2009$ & $2008-2009$ & $2008-2009$ & $2008-2009$ & $2008-2009$ \\
\hline $\begin{array}{l}\text { Lo uso más que } \\
\text { cualquier otro } \\
\text { medio de } \\
\text { comunicación }\end{array}$ & $62-71$ & $34-33$ & $16-17$ & $6-8$ & $7-10$ & $2-3$ \\
$\begin{array}{l}\text { Me permite } \\
\text { decidir lo que } \\
\text { veo, leo, escuch }\end{array}$ & $57-65$ & $40-41$ & $27-27$ & $17-23$ & $19-21$ & $4-7$ \\
$\begin{array}{l}\text { Es para } \\
\text { distraerme de } \\
\text { mis tareas } \\
\text { habituales }\end{array}$ & $53-52$ & $51-54$ & $25-27$ & $23-33$ & $10-14$ & $4-6$ \\
$\begin{array}{l}\text { Es más } \\
\text { confiable }\end{array}$ & $30-34$ & $36-33$ & $23-26$ & $13-20$ & $26-33$ & $4-4$ \\
\hline
\end{tabular}

Fuente: IAB (s. f.).

En el periodo 2008-2009, en México mejoró la percepción de los usuarios de Internet que fueron encuestados sobre los atributos de relación. Las excepciones se presentan en Internet (para distraerme de mis tareas habituales $-2 \%$ ), televisión (lo uso más que cualquier otro medio de comunicación $-1 \%$ ) y confiabilidad (-3\%).

\section{Los usuarios de Internet en el ambiente comunicativo de la web $\mathbf{2 . 0}$}

En los ambientes comunicativos de la web 2.0, los usuarios de Internet en México han descubierto formidables espacios de expresión. De acuerdo con los resultados que arrojó el reciente estudio de la IAB, 55\% de los usuarios de Internet en México han subido fotos o videos a sitios como Hi5, Facebook y YouTube. La tabla 12 
presenta los sitios predilectos de los usuarios de Internet en México para subir fotos o videos (tabla 12).

Tabla 12. En qué sitios han subido fotos o videos los usuarios de Internet en México

\begin{tabular}{|l|c|c|}
\hline Sitio & $\mathbf{2 0 0 8}$ & $\mathbf{2 0 0 9}$ \\
\hline Hi5 & 49 & 51 \\
Facebook & 18 & 44 \\
YouTube & 24 & 30 \\
My Space & 27 & 28 \\
Flickr & 25 & 16 \\
\hline
\end{tabular}

Fuente: IAB (s. f.).

De acuerdo con el referido estudio de la $I A B$, el 51\% de los usuarios de Internet en México se habían registrado en alguna red social en 2008. En 2009 el porcentaje de usuarios de Internet en México registrados en alguna red social se incrementó a 70\% (en un año el incremento registrado fue 19\%). La tabla 13 presenta las principales redes sociales en las que están registrados o participan los usuarios de Internet en México.

Tabla 13. Principales redes sociales en las cuales participan o están registrados los usuarios de Internet en México

\begin{tabular}{|l|l|l|}
\hline Sitio & $\mathbf{2 0 0 8}$ & $\mathbf{2 0 0 9}$ \\
\hline Hi5 & 58 & 66 \\
Facebook & 23 & 55 \\
MySpace & 34 & 34 \\
Windows Live & 31 & 30 \\
Sonico & 12 & 22 \\
Metro Flog & 11 & 13 \\
\hline
\end{tabular}

Fuente: IAB (s. f.).
Es posible anticipar que muy pronto Facebook desplazará a Hi5 como la red más popular entre los usuarios de Internet en México. En los estudios que se realicen durante 2010 sobre hábitos de los usuarios de Internet en México podremos confirmarlo.

\section{Conclusión}

Hasta 2009 las principales investigaciones relativas a la penetración y el impacto de Internet en México fueron realizados por organismos que representan los intereses comerciales de los grandes jugadores de la industria de Internet, como la Asociación Mexicana de Internet (Amipci) y la IAB. Actualmente, los estudios que ha realizado la Amipci sobre los hábitos de los usuarios de Internet en México representan la principal fuente de información para toda aquella persona interesada en analizar el desarrollo y el impacto de Internet en este país.

Un análisis longitudinal de los estudios realizados por la Amipci permite advertir algunas deficiencias metodológicas - cada año las encuestas son publicadas en los sitios web de las empresas e instituciones afiliadas a la Amipci y cualquier persona puede responderlas-. Además, en cada estudio se han registrado frecuentes modificaciones en las variables consideradas $\mathrm{y}$, por supuesto, cambios en algunos de los principales rangos utilizados, por ejemplo edad. En un principio los estudios eran realizados por la firma Select, la cual se prestó a realizar los cambios sugeridos por los directivos de Amipci sin oponer argumentos metodológicos. Los cambios respondían al propósito de "exagerar" la importancia y penetración de Internet en la sociedad.

En los años recientes los estudios realizados por la Amipci lamentablemente perdieron credibilidad. Los errores y las contradicciones fueron evidentes no solo para expertos en temas de Internet responsables de realizar la investigación sobre hábitos de los usuarios. Tal situación no 
conviene a los grandes jugadores de esta industria en México, ni a los investigadores dedicados al estudio de Internet, ni a los usuarios. Disponer de información dudosa no conviene a nadie.

Por esa sencilla razón -carecer de información fidedigna sobre el desarrollo de Internet en México- decidimos realizar las gestiones necesarias con Jeffrey Cole y el Centro para el Futuro Digital en la Universidad del Sur de California para asumir, a partir de 2009, la responsabilidad de realizar año a año el estudio que demanda el WIP.

Los resultados del primer estudio - pero principalmente el aprendizaje adquirido- fueron positivos. En este texto pretendimos presentar, además de algunos de los principales resultados que arrojó nuestro estudio, un panorama aproximado al estado de desarrollo de Internet en México. Estamos a punto de liberar los resultados que arrojó el estudio realizado a finales de 2009. La severidad de la crisis económica de 2009 afectó a la población muestra empleada en el primer estudio. Cerca del $20 \%$ de las personas que formaban parte de la muestra perdieron sus líneas telefónicas. Por supuesto, en una próxima colaboración para Palabra Clave deseamos dar a conocer los resultados que arrojó el segundo estudio.

\section{Referencias}

Amipci (2009). Estudio AMIPCI. Hábitos de los usuarios de Internet en México. Disponible en: http://www.amipci.org.mx/estudios/

e-Marketer (s. f.). Mexico Online. Disponible en http://www.slideshare.net/Engelnator/e-marketer-mexico-online-2009-presentation [Fecha de consulta: 22 de enero de 2010].

IAB (s. f.). Estudio de consumo de los medios digitales en México (Preparado por Millward Brown
México). Disponible en http://www.iabmexico. $\mathrm{com} / \mathrm{medioteca} / \mathrm{medio} \cdot \mathrm{php}$ ?id=211\&r=p [Fecha de consulta: 22 de enero de 2010].

IBOPE (2009). Anuario 2008-2009 Audiencias y Medios en México. México: IBOBE AGB.

INEGI (2010). Encuesta nacional sobre disponibilidad y uso de las tecnologías de la información en los hogares 2009. Disponible en http://www.inegi.org. $\mathrm{mx} / \mathrm{est} / \mathrm{contenidos/espanol/proyectos/metada-}$ tos/encuestas/endutih_2311.asp? s=est\&c=10850 [Fecha de consulta: 22 de enero de 2010].

Islas, O. et ál. (2000). Internet. El medio inteligente. México: Cecsa.

Islas, O. et ál. (2002). .com probado. México: Cecsa.

Levinson, P (1997). The soft age a natural history and future of the information revolution. London: Routeledge.

Levinson, P (2004). Cellphone: The Story of the World's Most Mobile Medium and How It Has Transformed Everything! New York: Palgrave Macmillan.

Universal McCann (2008). Power to the People: Social Media Tracker Wave 3,. Disponible en http://www.universalmccann.com/Assets/ wave_3_20080403093750.pdf [Fecha de consulta: 22 de enero de 2010].

WEF (2009). The Global Information Technology Report 2008-2009. Disponible en http://www.weforum.org/pdf/gitr/2009/Rankings.pdf [Fecha de consulta: 22 de enero de 2010].

World Internet Project-México (2010). Disponible en http://wipmexico.org/2201.html [Fecha de consulta: 22 de enero de 2010]. 